\title{
Entalpías de Inmersión de Carbones Activados en Soluciones Séricas de Carbamazepina
}

\author{
Natalia Bohórquez ${ }^{1}$, Liliana Giraldo ${ }^{2}$ y Juan C. Moreno-Piraján ${ }^{1 *}$ \\ (1) Universidad de los Andes, Facultad de Ciencias, Departamento de Química, Carrera 1 \\ No. 18 A 10, Bogotá-Colombia (e-mail: nbohorquez@uniandes.edu.co; jumoreno@uniandes.edu.co) \\ (2) Universidad Nacional de Colombia, Facultad de Ciencias, Departamento de Química, Ciudad \\ Universitaria, Carrera 30 No. 45 03, edificio 451, Bogotá-Colombia (e-mail: Igiraldogu@unal.edu.co)
}

*autor a quien debe ser dirigida la correspondencia

\section{Resumen}

Se determinan la entalpía de inmersión y la capacidad de adsorción de carbones activados en soluciones séricas de carbamazepina. Los carbones activados se modifican con soluciones de ácido nítrico de $40 \%$ y $65 \%$ a $303 \mathrm{~K}$, aumentando el contenido de sitios ácidos totales en la superficie. Se establecen relaciones entre el área superficial y la variación en la entalpía de inmersión en función del tratamiento de modificación y la cantidad adsorbida de carbamazepina. Se observa que para los sólidos obtenidos a partir de carbón mineral, la interacción está influenciada por características físicas y químicas de la superficie, en tanto que para los sólidos obtenidos a partir del carbón activado comercial se aprecia mayor interacción al aumentar el contenido de grupos ácidos. Los carbones activados con mayor absorción de carbamazepina son: los de origen mineral modificado, CAmi40 CAmi65, y carbón comercial modificado con ácido nítrico de mayor concentración, CAmk65.

Palabras clave: entalpías de inmersión, carbones activados, carbamazepina, modificación ácida

\section{Immersion Enthalpies of Activated Carbon in Carbamazepine Serum Solutions}

\begin{abstract}
The immersion enthalpy and the adsorption capacity of activated carbon in carbamazepine serum solutions are determined in this study. The activated carbon were modified with nitric acid solutions of 40 and $65 \%$ at $303 \mathrm{~K}$, increasing the content of the total acid sites on the surface. Relations between the superficial area and the immersion enthalpy variation in function of modification treatment and the adsorbed amount of carbamazepine. It was observed that for solids obtained from mineral coal, the interaction is influenced by the physical and chemical surface characteristics, while for solids obtained from the commercial activated carbon it was observed a greater interaction as the content of acid groups increases. The activated carbons with higher carbamazepine adsorption are: the modified mineral activated carbons, CAmi40, CAmi65 and the commercial carbon modified with nitric acid with a higher concentration, CAmk65.
\end{abstract}

Keywords: immersion enthalpy, activated carbon, carbamazepine, acid modification 


\section{INTRODUCCIÓN}

Como es bien conocido, el carbón activado es un material adsorbente que presenta un elevado y variado grado de porosidad, una considerable superficie interna y un cierto contenido de grupos químicos superficiales; estas características son las responsables de su alta capacidad de adsorción que se utiliza en aplicaciones en fase gaseosa y fase líquida. Su utilización como adsorbente versátil, se fundamenta en que el tamaño y distribución de sus poros en la estructura carbonosa pueden ser controlados para satisfacer las necesidades de adsorción y desorción de diferentes compuestos (Cyted, 2004).

En la medida en que el campo de aplicación del carbón se amplía, como en el caso de la adsorción y disponibilidad de compuestos de carácter farmacéutico, a la producción de las clásicas formas granulares y en polvo se ha unido la fabricación de fibras, telas, monolitos, entre otros, materiales en los que se puede conseguir, de acuerdo al método de preparación, una determinada distribución de la porosidad y/o de la naturaleza química de la superficie (Cartula et al, 1998).

En la adsorción de compuestos orgánicos uno de los aspectos que tiene mayor influencia es la química de la superficie (McGuire y Suffet, 1983), la que depende del contenido de heteroátomos, principalmente grupos con oxígeno; que determinan en los carbones activados la carga de la superficie, la hidrofobicidad y la densidad electrónica de las capas grafénicas. La carga superficial depende del $\mathrm{pH}$ del medio y de las características de la superficie del carbón, la carga superficial negativa proviene de la disociación de los grupos superficiales de carácter ácido como los grupos carboxílicos y fenólicos. La carga positiva, no se atribuye a grupos superficiales tan específicos y puede estar constituida por grupos, de carácter básico como pironas o cromenos o por la existencia de zonas en la superficie ricas en electrones $\pi$ que se comportan como bases de Lewis (MorenoCastilla, 2004).

La existencia de grupos químicos en la superficie de los carbones activados hace que las partículas en solución acuosa presenten una carga eléctrica que influye en los procesos de adsorción; la carga eléctrica se establece con el punto isoeléctrico, PIE, o el punto de carga cero, PZC, en el primero se cuantifica fundamentalmente la carga de la superficie más externa de las partículas y el segundo mide la carga superficial total (Radovic et al., 2000).

La adsorción sobre carbón activado de algunos medicamentos sintéticos se estudia desde el punto de vista de remover del cuerpo los excesos que se pueden presentar cuando se tiene un uso prolongado de dicho medicamento, otro uso frecuente del carbón activado se refiere a la adsorción de toxinas que se encuentran en la sangre. Como ejemplo del primer tipo de aplicación, se puede citar la carbamazepina, $\mathrm{C}_{15} \mathrm{H}_{12} \mathrm{~N}_{2} \mathrm{O}$ es un compuesto tricíclico dibenzazepina derivado del imino estilbeno (Suwalsky et al., 2006; Tayel et al., 2007), que es uno de los medicamentos de mayor consumo para el tratamiento de enfermedades epilépticas, diversos estados psiquiátricos tales como el síndrome de abstinencia alcohólica, la profilaxis de los estados maniacodepresivos y en alteraciones de tipo neurológico; de igual manera se utiliza como analgésico; este medicamento puede ocasionar serias alteraciones en el organismo si se supera la concentración terapéutica de $10 \mu \mathrm{gmL}^{-1}$, y además producir una serie de efectos secundarios por la acumulación progresiva de sus componentes (Neuvonen y Clonen,1980; Gaudreault, 2005); así mismo, dependiendo de condiciones externas sus suspensiones acuosas pueden producir compuestos secundarios (Tian et al., 2006).

En este trabajo se modifican, por tratamiento con ácido nítrico, dos tipos de carbón activado: uno comercial y otro preparado a partir de un carbón mineral subituminoso. Los carbones activados obtenidos se usan como un tratamiento para reducir los niveles séricos de carbamazepina. Como la adsorción de la carbamazepina depende tanto de las propiedades texturales como químicas de la superficie, se determinan para los materiales el área superficial BET, la distribución de tamaño de poro, la acidez y la basicidad total y los espectros infrarrojos de los carbones activados originales y los modificados. La variación entálpica producida por la interacción entre el adsorbato y adsorbente, permite conocer la intensidad de la interacción y por tanto se tiene como objetivo realizar calorimetrías de inmersión, que como resultado lleven a obtener un valor característico de la interacción. Por último se establece mediante una filtración en una columna de lecho empacado, la capacidad de adsorción de carbamazepina de cada una de las muestras. 


\section{METODOLOGÍA}

\section{Obtención de los carbones activados.}

Se utilizan dos tipos de carbón activado para realizar este estudio: una muestra de carbón activado comercial Merck® en polvo, CAmk, y una muestra de carbón activado mineral, CAmi, con granulometría de $1.4 \mathrm{~mm}$, obtenido a partir de carbón subituminoso preparado por carbonización en atmósfera de $\mathrm{N}_{2}$ a $1273 \mathrm{~K}$ por 2 horas, seguido de una activación con vapor de agua a $1123 \mathrm{~K}$ durante 9 horas, después de la cual se consigue un $48 \%$ de burn-off.

\section{Modificación química de los carbones activados con soluciones de ácido nítrico.}

Las muestras de carbón activado se someten a tratamientos con soluciones al 40 y $65 \%$ de $\mathrm{HNO}_{3}$, para modificar los grupos químicos sobre la superficie y, establecer como tales modificaciones influyen en las interacciones del carbón activado con la carbamazepina en soluciones de suero. Los carbones activados se sumergen en la solución correspondiente de ácido por un periodo de 24 horas, en agitación constante a una temperatura de $303 \mathrm{~K}$; a continuación se filtran al vacío las muestras y se lavan con agua desionizada hasta pH neutro. Los carbones modificados, CAmk40, CAmk65, CAmi40 y CAmi65, se almacenan en atmósfera de nitrógeno hasta su utilización.

\section{Determinación de isotermas de $N_{2}$.}

Se determinan las isotermas de adsorción de nitrógeno a 77K, con un sortómetro Quantachrome Autosorb3B a los carbones activados de partida y a los modificados y se calcula el área superficial y el volumen de poro.

\section{Determinación de los grupos ácidos y básicos totales.}

Se determinan la acidez y la basicidad total por medio de titulaciones (Boehm, 1966) que se realizan con un equipo automático TA20 Plus Titro Line Alpha Plus marca SCHOTT. Se pesa aproximadamente $1.000 \mathrm{~g}$ de cada sólido, se coloca en un frasco de vidrio con tapa esmerilada con $50.0 \mathrm{~mL}$ de hidróxido de sodio, $\mathrm{NaOH}, 0.1 \mathrm{~N}$; una cantidad similar se coloca en un recipiente con $50.0 \mathrm{~mL}$ de ácido clorhídrico, $\mathrm{HCl}, 0.1 \mathrm{~N}$. Las soluciones se tapan, se dejan a temperatura constante de $298 \mathrm{~K}$, durante 5 días agitándose continuamente mediante agitación magnética. Una vez pasados los 5 días se toman muestras de $10.0 \mathrm{~mL}$ de cada una de las soluciones y cada una se titula con la solución estándar de hidróxido de sodio o ácido clorhídrico según sea el caso.

Para verificar cambios en los grupos químicos de la superficie de los carbones activados, se determinan los espectros infrarrojos en un espectrómetro infrarrojo Thermo Nicolet por transformadas de fourier FTIR marca Nexos, con celda de reflactancia difusa. Se utilizaron aproximadamente $40 \mathrm{mg}$ de cada muestra de carbón activado.

Determinación de la variación en la entalpía de inmersión de los carbones activados en suero y soluciones séricas de carbamazepina.

En este trabajo se realizan determinaciones de la entalpía de inmersión de los carbones activados, de partida y modificados, en suero y soluciones séricas de carbamazepina de $500 \mu \mathrm{gmL}^{-1}$ para determinar las interacciones energéticas cuando el carbón activado está en contacto con estas soluciones. Para determinar los calores de inmersión se usa un microcalorímetro de conducción de calor con una celda calorimétrica en acero inoxidable (Giraldo, et. al., 2002; Ladino, et. al., 2005). Se colocan en la celda $10 \mathrm{~mL}$ de la solución a utilizar, que se han mantenido en un termostato a $298 \mathrm{~K}$; se pesa una muestra de carbón activado del orden de $100 \mathrm{mg}$ y se coloca dentro de la celda calorimétrica en una ampolleta de vidrio, se ensambla el microcalorímetro. Cuando el equipo alcanza una temperatura de alrededor $298 \mathrm{~K}$, se inicia el registro de potencial de salida por un periodo de aproximadamente 15 minutos tomando lecturas de potencial cada 20 segundos, se procede a realizar el rompimiento de la ampolleta de vidrio, se registra el efecto térmico generado y se continua con las lecturas de potencial por aproximadamente 15 minutos más, finalmente se calibra eléctricamente. 
Determinación de la adsorción de carbamazepina en los carbones activados en sistema de flujo.

La adsorción de la carbamazepina se realiza en una columna de vidrio de $4.2 \mathrm{~cm}$ de longitud y $3.4 \mathrm{~cm}$ de diámetro, con un vidrio sinterizado en el fondo que soporta el CA e impide la salida del mismo con el efluente. El flujo se mantuvo en continuo a $0.8 \mathrm{~mL} \mathrm{~min}^{-1}$ y se alimenta la columna con una bomba peristáltica Chromatography Puma B-688 marca Büchi. La longitud del lecho empacado es de $0.7 \mathrm{~cm}$, que equivale a 1.0 $\mathrm{g}$ de carbón. El fluido a filtrar consiste en una solución de suero fisiológico con una concentración de carbamazepina de $12 \mu \mathrm{gmL}^{-1}$. Se toman alícuotas cada 5 minutos y se mide la absorbancia de las mismas a $290 \mathrm{~nm}$ con un espectrofotómetro UV, Thermo Spectronic Genesys 5, cuya concentración se obtiene mediante una calibración previa.

\section{RESULTADOS Y DISCUSIÓN}

A las muestras de carbón activado se les determinan sus propiedades superficiales por medio de las isotermas de $\mathrm{N}_{2}$ a $77 \mathrm{~K}$, que permiten conocer el área superficial y el volumen total de poro, y las características de acidez y basicidad total que son indicativos de los grupos químicos en la superficie. En la Tabla 1 se presentan los resultados obtenidos.

Tabla 1: Características superficiales de los carbones activados originales y modificados

\begin{tabular}{|c|c|c|c|c|}
\hline Carbón activado & $\begin{array}{c}\text { Área superficial } \\
\left(\mathrm{m}^{2} \mathrm{~g}^{-1}\right)\end{array}$ & $\begin{array}{c}\text { Volumen de } \\
\text { poro } \\
\left(\mathrm{cm}^{3} \mathrm{~g}^{-1}\right)\end{array}$ & $\begin{array}{c}\text { Acidez total } \\
\left(\mathrm{meqg}^{-1}\right)\end{array}$ & $\begin{array}{c}\text { Basicidad total } \\
\left(\mathrm{meqg}^{-1}\right)\end{array}$ \\
\hline Cami & 980 & 0.48 & 0.36 & 0.43 \\
\hline CAmi40 & 1025 & 0.48 & 0.49 & 0.30 \\
\hline CAmi65 & 942 & 0.45 & 0.54 & 0.28 \\
\hline Camk & 622 & 0.36 & 0.44 & 0.35 \\
\hline CAmk40 & 609 & 0.34 & 0.47 & 0.32 \\
\hline CAmk65 & 576 & 0.31 & 0.52 & 0.31 \\
\hline
\end{tabular}

Se puede observar que el tratamiento con $\mathrm{HNO}_{3}$ modifica la superficie de los carbones activados iniciales comercial Merck y mineral, CAmk y CAmi, tanto en sus propiedades físicas como químicas; en general se aumenta el contenido de sitios ácidos totales, se disminuye el contenido de sitios básicos totales y el aumento en los primeros es mayor cuando la solución de ácido nítrico es más concentrada, del $65 \%$. En cuanto a los valores de área superficial, en la mayoría de los casos, éste disminuye debido a la inclusión de grupos oxigenados, pero se observa que no ocurre así con el carbón activado mineral tratado con solución de $\mathrm{HNO}_{3}$ al $40 \%$, CAmi40, en el que se presenta un ligero aumento en el área superficial.

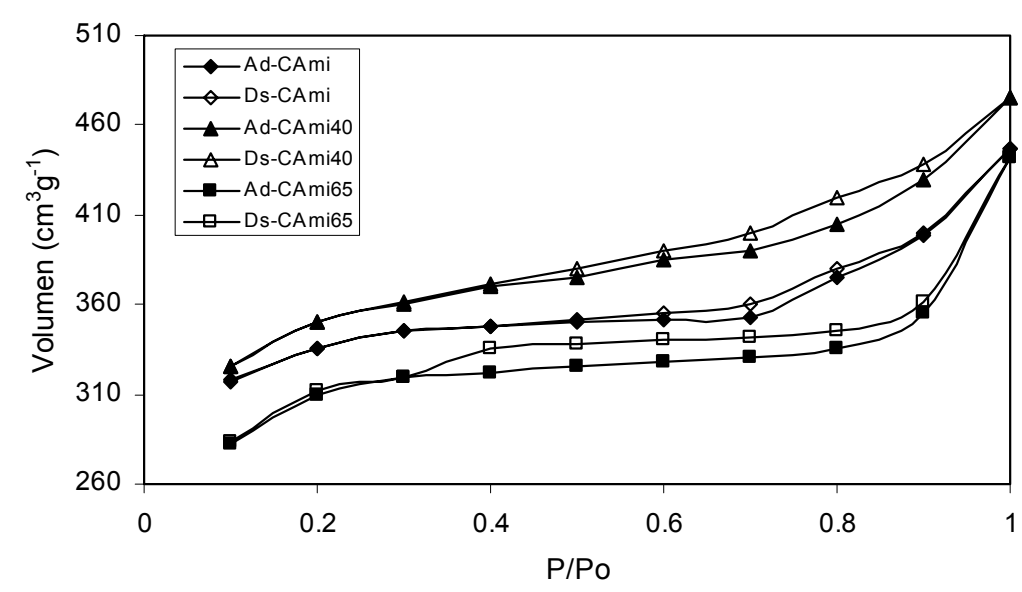

Fig. 1: Isotermas de adsorción de $\mathrm{N}_{2}$ para los carbones activados de origen mineral, CAmi 
En las Figuras 1 y 2 se muestran las isotermas de adsorción y desorción de las series de carbones activados, CAmi y CAmk respectivamente; en todos los casos las isotermas presentan ciclos de histéresis, sin embargo se observan las diferencias entre los dos materiales por la forma que tienen las isotermas y las cantidades que se adsorben para los diferentes valores de presión parcial, P/Po. Inicialmente, a presiones relativas bajas la adsorción aumenta y luego se mantiene constante a presiones medias, esto corresponde a la isoterma de tipo I de acuerdo a la clasificación de la IUPAC (Rodríguez-Reinoso, 1998) que corresponde a la adsorción

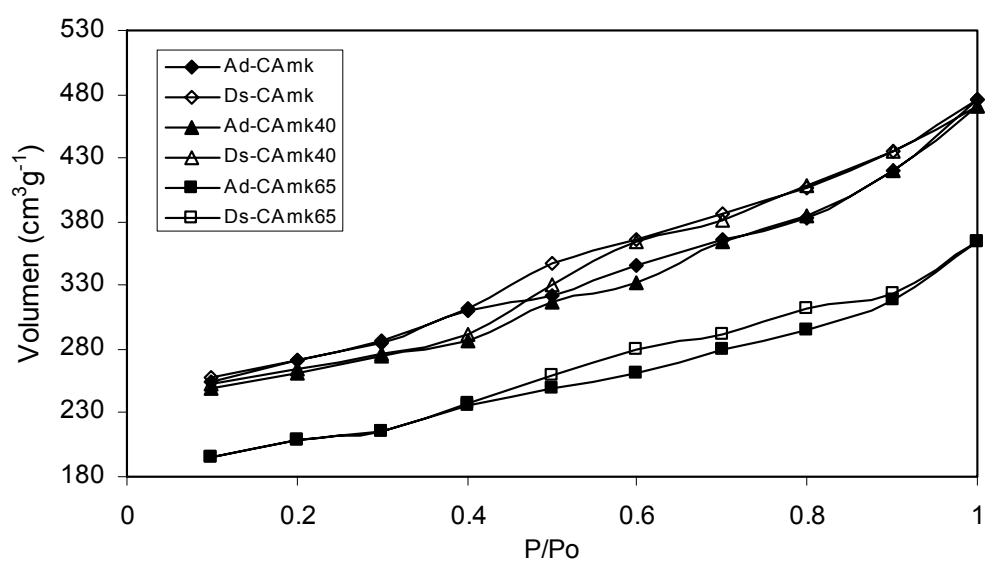

Fig. 2: Isotermas de adsorción de $\mathrm{N}_{2}$ para los carbones activados obtenidos a partir del cabón activado comercial Merck, CAmi

sobre sólidos microporosos, y posteriormente se presenta una leve condensación que produce los ciclos de histéresis. En la Figura 1 se observa una adsorción más alta de $\mathrm{N}_{2}$ para el carbón activado Cami40 y la menor para el Cami65; en la Figura 2 se presenta la mayor adsorción para la muestra CAmk y la menor para Camk65 , lo anterior se refleja en los valores obtenidos para el área superficial.

Con el propósito de comprobar la modificación en la química superficial de las muestras, se determinan los espectros FTIR para las seis muestras de carbón activado, los cuales presentan similitudes en la aparición de las bandas y variación en la intensidad de los picos para los carbones activados modificados. En las Figuras 3 y 4 se muestran los espectros infrarrojo para las muestras Cami y Cami40 respectivamente. Para estos carbones activados se encuentran valores de acidez total de 0.36 meqg $^{-1}$ para Cami y de $0.49 \mathrm{meqg}^{-1}$ para el Cami40, que indica la influencia del tratamiento con el ácido nítrico y que se puede verificar en la intensidad de las bandas que se muestran en los espectros infrarrojo de cada muestra.

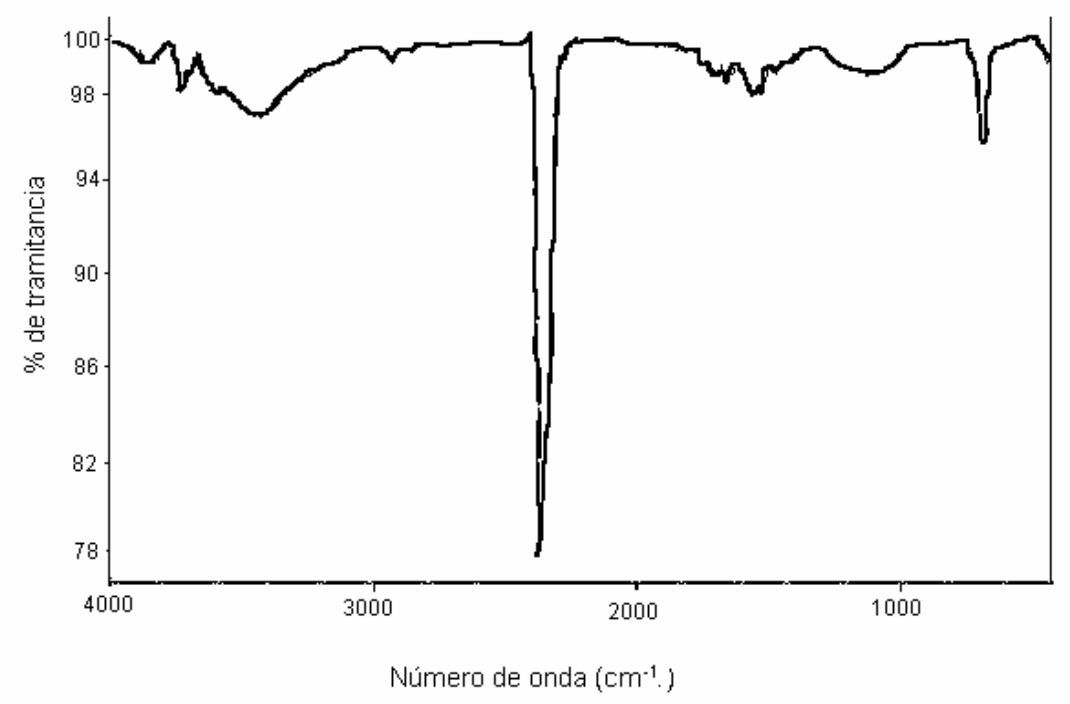

Fig. 3: Espectro FTIR para el carbón activado mineral, CAmi 


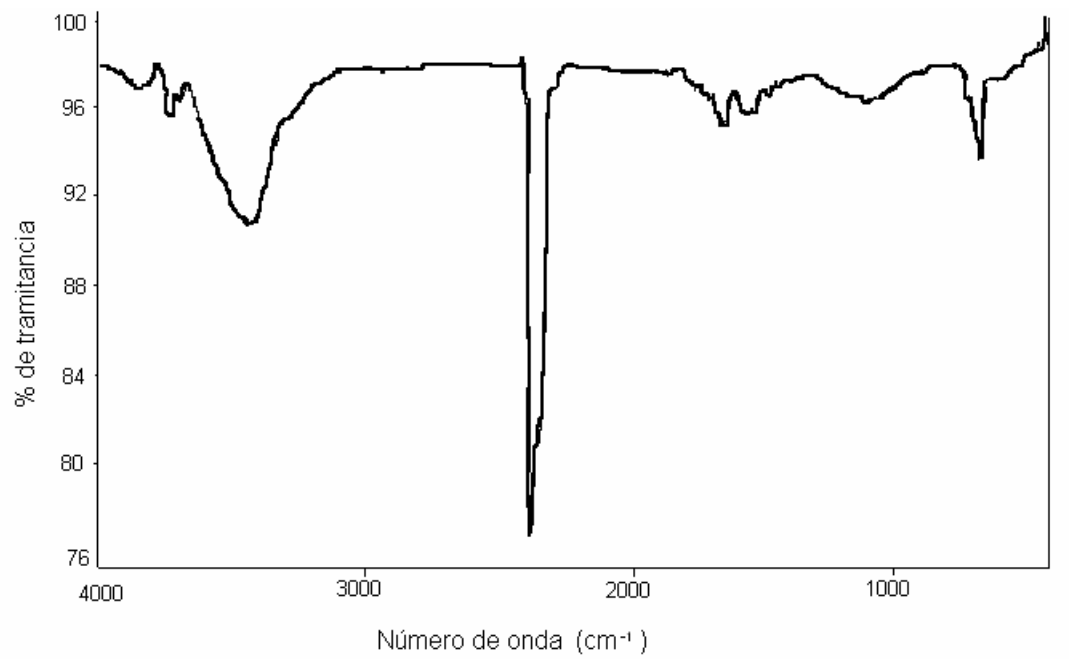

Fig. 4: Espectro FTIR para el carbón activado mineral modificado con solución $\mathrm{HNO}_{3}$ al $40 \%$, Cami40

Alrededor de $2300 \mathrm{~cm}^{-1}$ aparece un pico significativo en todas las muestras y con una intensidad similar que corresponde a la presencia de $\mathrm{CO}_{2}$ en las muestras (Chen et.al., 2003). Aparece un pico para números de onda entre 3200 y $3500 \mathrm{~cm}^{-1}$ que corresponde a la vibración de alargamiento del grupo funcional O-H; que se puede relacionar con otro pico ubicado en $670 \mathrm{~cm}^{-1}$ el cual corresponde a la flexión del enlace C-C del grupo funcional Aryl-O-H. Seguido a éstos, en algunos de los espectros, aparecen picos de pequeña intensidad a $2900 \mathrm{~cm}^{-1}$ que corresponden a los grupos funcionales $\mathrm{C}-\mathrm{H},-\mathrm{CH}_{2},-\mathrm{CH}_{3}$.

Se observan además, una serie de picos entre los números de onda 1400 y $1700 \mathrm{~cm}^{-1}$, de moderada intensidad, que pueden atribuir alargamientos de los grupos funcionales de tipo, $\mathrm{C}=\mathrm{O}$ y $\mathrm{C}=\mathrm{C}$, que pueden corresponder a la presencia de cetonas, ésteres, aldehidos y ácidos carboxílicos (Shin et al., 1997). En 1060 $\mathrm{cm}^{-1}$ se observa un pico que corresponde al grupo funcional C-O. En la Tabla 2 se presentan los grupos funcionales correspondientes para cada pico y la intensidad de cada uno, en porcentaje de transmitancia, para realizar la comparación entre los carbones activados modificados con respecto al espectro del carbón activado de partida.

Tabla 2: Resumen de la información obtenida de los espectros infrarrojo determinados para los carbones activados

\begin{tabular}{|c|c|c|c|c|c|}
\hline \multirow{4}{*}{ Muestra } & \multicolumn{5}{|c|}{ Banda correspondiente a un qrupo funcional } \\
\hline & Aryl-OH & $\mathrm{C}-\mathrm{O}$ & $\mathrm{C}=\mathrm{O}, \mathrm{C}=\mathrm{C}$ & $\begin{array}{c}\mathrm{C}-\mathrm{H}, \mathrm{CH} 2, \\
\mathrm{C}-\mathrm{H} 3\end{array}$ & $-\mathrm{OH}$ \\
\hline & $\begin{array}{l}670 \\
\left(\mathrm{~cm}^{-1}\right)\end{array}$ & $\begin{array}{l}1060 \\
\left(\mathrm{~cm}^{-1}\right)\end{array}$ & $\begin{array}{c}1400-1700 \\
\left(\mathrm{~cm}^{-1}\right)\end{array}$ & $\begin{array}{l}2900 \\
\left(\mathrm{~cm}^{-1}\right)\end{array}$ & $\begin{array}{c}3200-3500 \\
\left(\mathrm{~cm}^{-1}\right)\end{array}$ \\
\hline & \multicolumn{5}{|c|}{$\%$ de tramitancia } \\
\hline CAmi & 95 & 99 & 98 & 99 & 97 \\
\hline CAmi40 & 93 & 97 & 95 & - & 94 \\
\hline CAmi65 & 93 & 94 & 93 & - & 91 \\
\hline Camk & 97 & 98 & 97 & 98 & 95 \\
\hline Camk40 & 95 & 97 & 96 & - & 93 \\
\hline Camk65 & 95 & 96 & 95 & - & 91 \\
\hline
\end{tabular}

Los resultados obtenidos en los espectros infrarrojo, muestran que se presenta una variación en la intensidad de los picos que aparecen para determinados números de onda asociados con grupos funcionales que contienen oxígeno y que pueden interactuar con la carbamazepina; sin embargo el cambio en la intensidad, 
como se observa por los porcentajes de tramitancia, es poco ya que el tratamiento con las soluciones de ácido nítrico se realiza en unas condiciones de temperatura moderada de $303 \mathrm{~K}$, y si se compara con otros trabajos (Li et al., 2003) en los que se usan temperaturas mayores, se encuentra que la inclusión de grupos oxigenados es menor.

Para conocer la intensidad energética de la interacción entre los carbones activados y el sistema de interés, que es la solución sérica de carbamazepina, se determina la entalpía de inmersión que es una medida del calor total generado por el contacto del sólido con la solución líquida, que involucra por tanto la interacción con la superficie física como química del carbón y de las sustancias que se encuentran presentes en el líquido. En la Figura 5, se presenta un termograma típico de potencial en función del tiempo en el que se observa la presencia de tres picos: el primero corresponde a la inmersión del CAmi en suero y los otros dos se deben a la calibración eléctrica en la que se suministra al sistema calorimétrico una cantidad conocida de trabajo eléctrico.

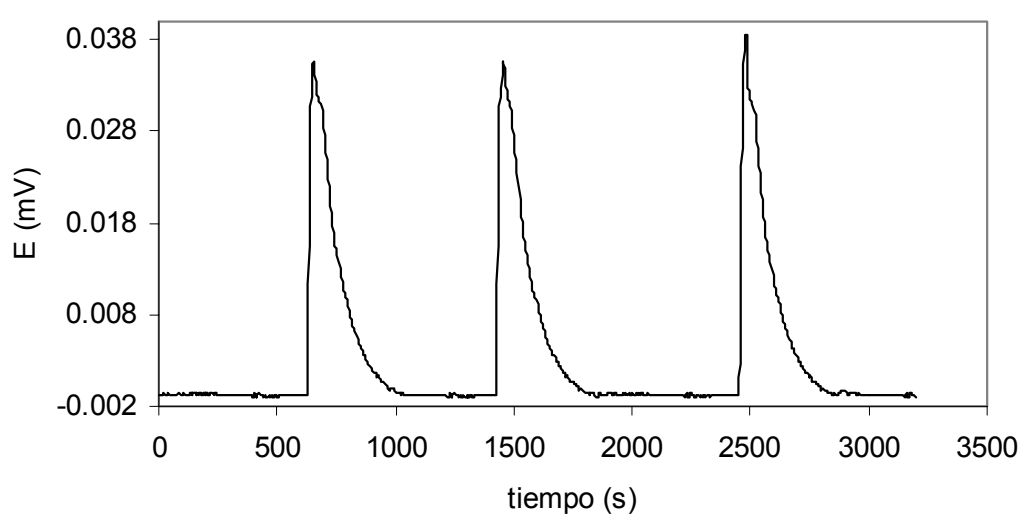

Fig. 5: Termograma obtenido en la inmersión del carbón activado mineral, CAmi, en suero

Se determina la entalpía de inmersión de las muestras de carbón activado en suero y en solución sérica de carbamazepina; y se calcula el cambio de entalpía neta, $\Delta$ Hinm neta, que corresponde únicamente a la interacción entre la carbamazepina y el carbón activado, y se obtiene de la diferencia entre el resultado obtenido para la variación de la entalpía de inmersión del carbón activado en la solución de carbamazepina y la variación de la entalpía de inmersión del mismo carbón activado con el suero (López et al., 1999); para esto se utiliza la siguiente ecuación:

$\Delta H_{\text {inm }}(\text { Carbamazepina })_{n e t o}=\Delta H_{\text {inm }}($ suero + carbamazepina $)-\Delta H_{\text {inm }}($ suero $)$

Tabla 3: Determinación de la entalpía de inmersión de los carbones activados en suero y solución sérica de carbamazepina

\begin{tabular}{|c|c|c|c|}
\hline Carbon activado & $-\Delta$ Hinm en suero & $\begin{array}{c}-\Delta \text { Hinm neta } \\
\text { carbamazepina } \\
\left(\mathrm{Jg}^{-1}\right)\end{array}$ & $\begin{array}{c}\text { Cantidad adsorbida } \\
\text { de carbamazepina } \\
\mathbf{X}\end{array}$ \\
\hline CAmi & 42.35 & 16.92 & 0.10 \\
\hline Cami40 & 45.23 & 20.56 & 0.11 \\
\hline Cami65 & 46.84 & 17.84 & 0.10 \\
\hline CAmk & 28.12 & 12.26 & 0.09 \\
\hline Camk40 & 30.27 & 15.16 & 0.09 \\
\hline Camk65 & 31.32 & 16.43 & 0.10 \\
\hline
\end{tabular}

En la Tabla 3 se presentan los resultados obtenidos para la entalpía de inmersión, $\Delta$ Hinm en $\mathrm{Jg}^{-1}$; para todas las muestras de carbón activado en suero, el resultado de la entalpía neta para la carbamazepina, $\Delta$ Hinm neta en $\mathrm{Jg}^{-1}$ y la cantidad de carbamazepina adsorbida por gramo de carbón activado, $\mathrm{X}$ en $\mathrm{mgg}^{-1}$, durante la 
experiencia calorimétrica y que se determina por espectrofotometría UV, una vez se realiza la inmersión del carbón activado y la calibración eléctrica del sistema .

De los resultados se observa que se presenta un efecto calorimétrico mayor para la serie de carbones activados provenientes del carbón mineral, tanto para la entalpía de inmersión en suero como el cambio neto de entalpía para la interacción del sólido con la carbamazepina, lo que indica que estas muestras poseen características que favorecen la adsorción del compuesto de interés y esto se refleja en las cantidades adsorbidas del mismo. La serie de carbones activados provenientes del carbón activado comercial Merck, aunque presentan valores de entalpía menores tiene buena capacidad para la adsorción de la carbamazepina, alrededor de 10 por ciento menores con respecto a la muestra CAmi40. Los resultados muestran que la interacción se favorece con el tratamiento ácido de las muestras de carbón activado.

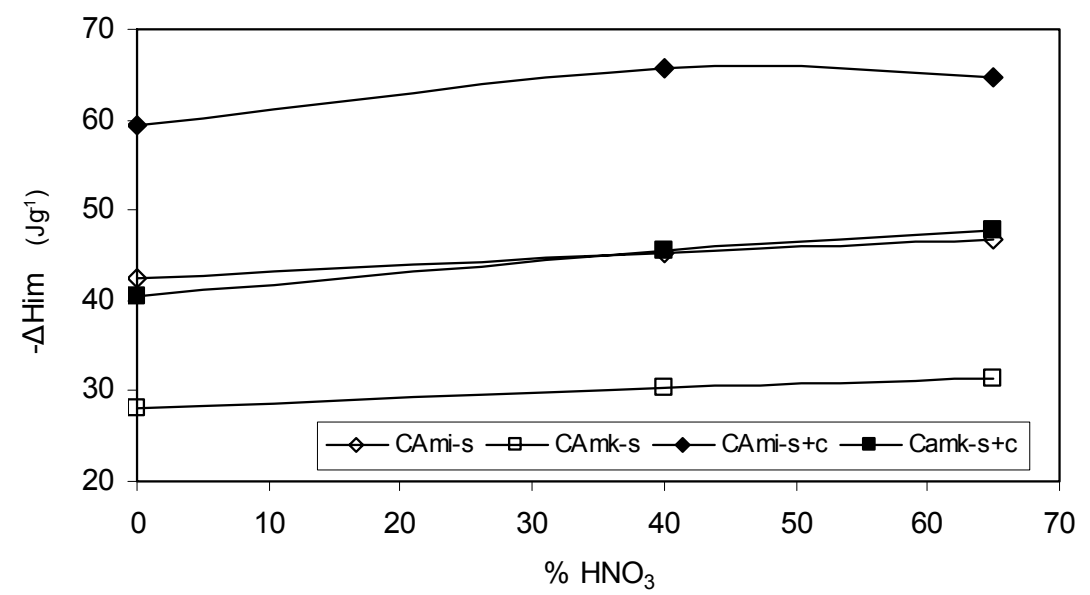

Fig. 6: Entalpía de inmersión de los carbones activados en suero y solución sérica de carbamazepina.

En la Figura 6 se muestra la relación que existe entre la entalpía de inmersión de los carbones activados en suero y en la solución sérica de carbamazepina en función del tratamiento ácido que se realiza; en ésta se observa que el efecto entálpico es mayor cuando las muestras se ponen en contacto con la solución de $500 \mathrm{\mu gmL}^{-1}$ de carbazepina, rombos y cuadros llenos, que cuando la inmersión se realiza en el suero como líquido calorimétrico de comparación, rombos y cuadros vacíos. La entalpía de inmersión tanto en suero como en la solución aumenta cuando sobre el carbón activado se realiza la oxidación de la superficie, excepto en el caso del CAmi65 para el cual se presenta una entalpía neta de carbamazepina menor que cuando el carbón mineral se trata con la solución de ácido nítrico al $40 \%$. La serie de carbones activados obtenidos del carbón mineral tiene una mayor interacción, hecho que se refuerza con los valores obtenidos para el área superficial que son mayores para esta serie y se observa en la Figura 6 que la inmersión de las muestras CAmi en solución sérica de carbamazepina presenta el efecto entálpico más fuerte.

Las Figuras 7 y 8 muestran la relación entre la entalpía neta de carbamazepina y el área superficial en función de la concentración de ácido nítrico usado para la modificación, para la serie de carbones activados CAmi y CAmk respectivamente. En la Figura 7 se puede apreciar que la entalpía neta en carbamazepina y el área superficial tienen la misma tendencia con respecto al tratamiento realizado al carbón activado de origen mineral; para el carbón activado mineral sin tratamiento ácido, CAmi, el valor del área superficial es de $980 \mathrm{~m}^{2} \mathrm{~g}^{-1}$, para la muestra que se trata con solución de ácido nítrico al $40 \%$, CAmi 40 , se presenta un aumento moderado en el valor del área superficial del cambio en la entalpía neta del soluto y finalmente para la muestra tratada con la solución del mismo ácido al $65 \%$ se obtiene el valor de área superficial más bajo. Con respecto a los resultados obtenidos para la entalpía neta de carbamazepina el menor valor se presenta para la muestra CAmi y el más alto corresponde a la muestra CAmi40.

En la Figura 8, que presenta los datos para la serie de los carbones activados obtenidos a partir del carbón activado comercial, CAmk, el comportamiento es diferente si se compara con la gráfica anterior, para estas 
muestras el área superficial disminuye con el tratamiento con ácido nítrico aunque dicha disminución es moderada, del orden de $40 \mathrm{~m}^{2} \mathrm{~g}^{-1}$, en tanto que la entalpía neta de carbamazepina aumenta con el tratamiento hecho sobre el carbón activado y también lo hace la cantidad de carbamazepina que se retiene en el sólido.

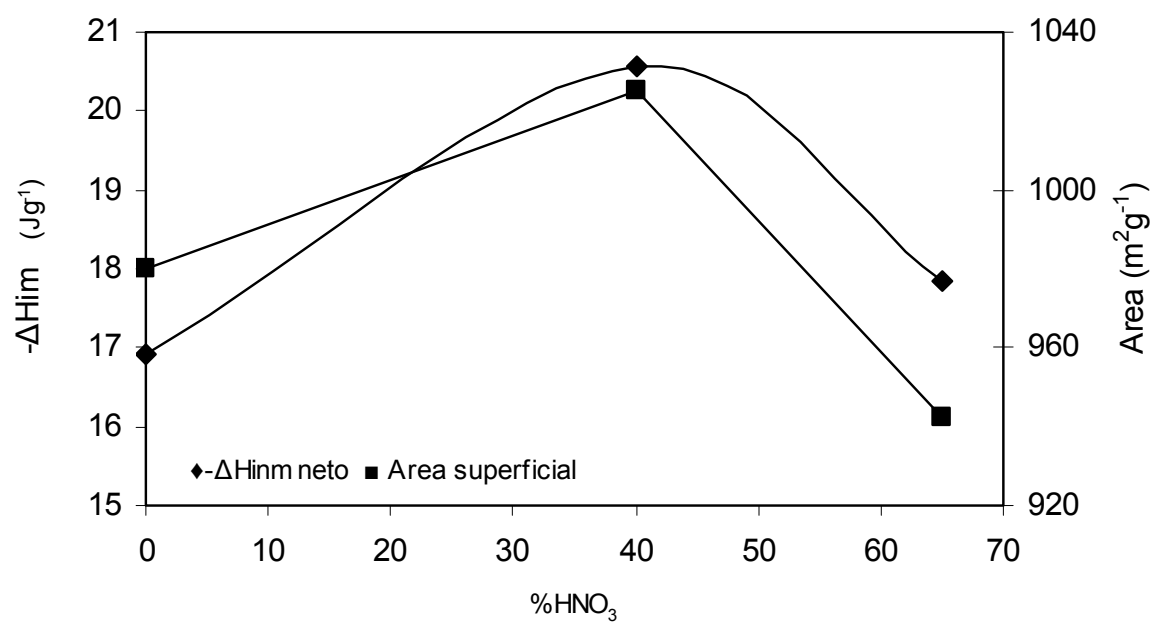

Fig. 7: Variación de la entalpía neta de carbamazepina y área superficial del carbón activado con respecto al tratamiento usado en la modificación superficial del carbón activado mineral

Los resultados muestran para la serie CAmi que la adsorción de la carbamazepina está influenciada por el área superficial más que por el contenido de sitios ácidos totales; mientras que para la serie CAmk aunque los valores de área superficial disminuyen, tal como ocurre en otros trabajos en los que se modifica la superficie química (Dastgheib y Karanfil 2004; Raymundo et al., 2003), el contenido de grupos ácidos totales aumenta y esto favorece la adsorción de la carbamazepina.

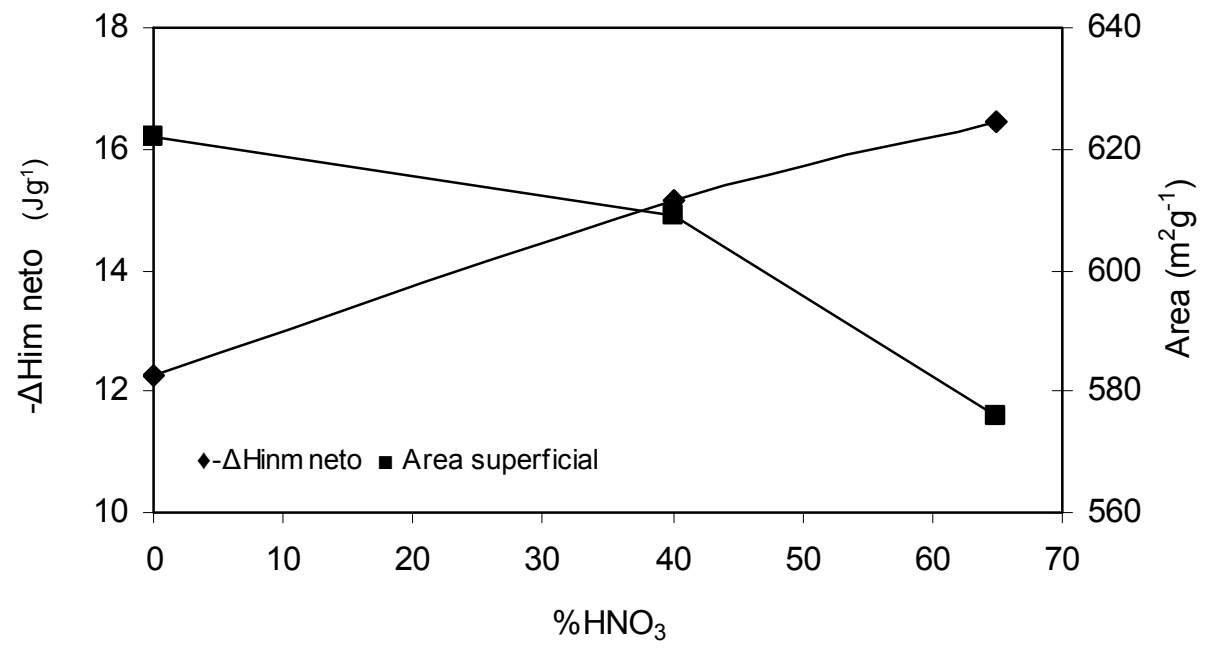

Fig. 8: Variación de la entalpía neta de carbamazepina y área superficial con respecto al tratamiento usado en la modificación superficial del carbón activado comercial Merck

Como se observa que la cantidad del soluto que se adsorbe se relaciona con la variación en la entalpía neta de interacción de la carbamazepina y que a su vez dicho valor de la variación en la entalpía es constituyente aditivo de la entalpía de inmersión de los carbones activados en las soluciones séricas del mismo; se establecen relaciones entre la entalpía neta de carbamazepina y los valores del área superficial de los carbones en función de la cantidad adsorbida de carbamazepina en el periodo en que se realiza la experiencia calorimétrica. Las Figuras 9 y 10 muestran los comportamientos para la serie CAmi y CAmk respectivamente. 


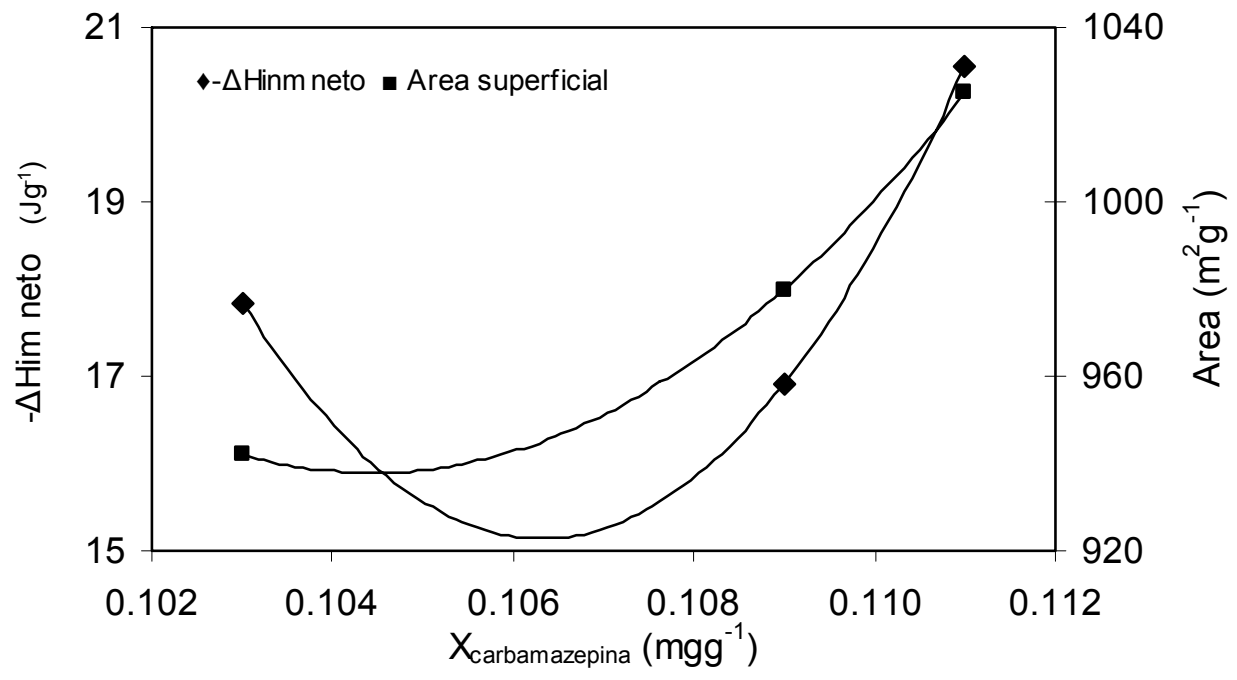

Fig. 9: Variación de la entalpía neta de carbamazepina y área superficial en función de la cantidad adsorbida de carbamazepina en la serie de carbones activados CAmi

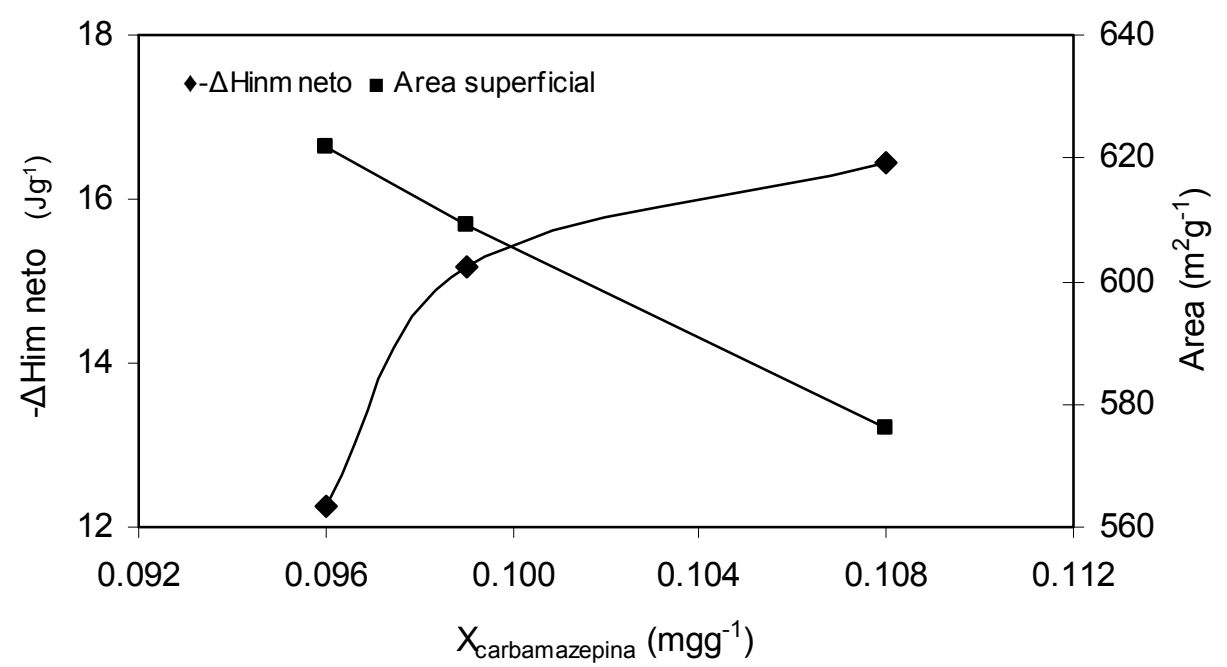

Fig. 10: Variación de la entalpía neta de carbamazepina y área superficial en función de la cantidad adsorbida de carbamazepina en la serie de carbones activados CAmk

Nuevamente se observa que la serie de carbones activados CAmi, tiene un comportamiento similar al relacionar la variación de la entalpía neta de carbamazepina en función de la cantidad adsorbida de ésta y el área superficial de los sólidos también en función de la cantidad adsorbida de carbamazepina, que se muestra en la Figura 9, y se ajusta a una tendencia de segundo orden en la que la muestra CAmi40 presenta los mayores valores para las tres características que se relacionan. En la Figura 10, el área superficial muestra una tendencia lineal con pendiente negativa, es decir la cantidad adsorbida de carbamazepina es mayor para el carbón activado CAmk65, que presenta el valor más bajo de área superficial y el contenido de acidez total mayor; en cuanto a la variación en la entalpía neta se observa que aumenta al aumentar la cantidad adsorbida de soluto.

Por último, como el uso del carbón activado para el tratamiento de la desintoxicación ha sido cuestionado, ya que algunos estudios muestran que la efectividad de éste disminuye significativamente luego de transcurridos 30 minutos de la ingesta de la sustancia tóxica (Krenzelok, 2002); se determina la cantidad adsorbida de carbamazepina en un gramo de carbón activado cuando una solución con una concentración de $5.0 \times 10^{-5} \mathrm{M}$ de carbamazepina se hace fluir a través de éste a una velocidad de $0.8 \mathrm{mLmin}^{-1}$ durante dos horas y establecer la disminución en la concentración 
cuando han transcurrido 20 minutos tiempo en el que el carbón activado debe ser efectivo para retirar la carbamazepina. En la Figura 11 se presenta los resultados obtenidos para las muestras de carbón activado usadas en este trabajo.

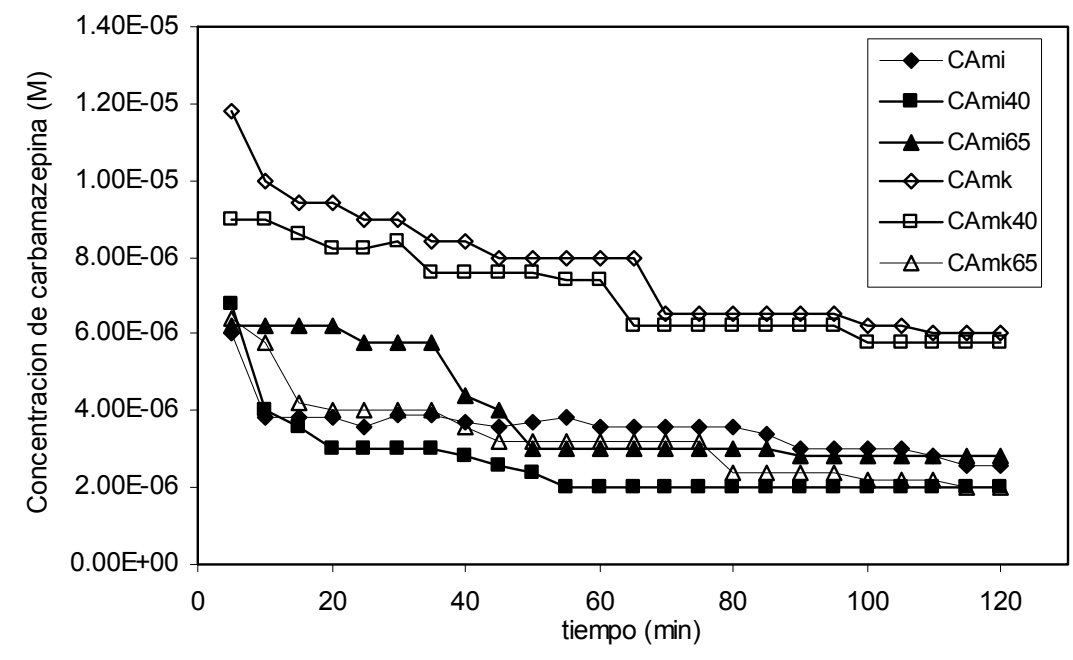

Fig. 11: Capacidad de adsorción de carbamazepina en las series de carbón activado CAmi y CAmk

En la Figura 11 se observa que la concentración del efluente en los primeros 20 min disminuye significativamente en todos los casos. Las muestras que presentan la mayor adsorción de carbamazepina en este tiempo son: la muestra de carbón mineral modificado con solución de ácido nítrico al 40\%, CAmi40, la de carbón activado comercial Merck modificado con solución de ácido nítrico al 65\%, CAmk65 y la de carbón activado mineral sin modificar CAmi. El valor promedio de concentración de carbamazepina en el efluente durante este tiempo para las muestras de carbón anteriormente mencionadas, fue igual a $3.46 \times 10^{-6} \mathrm{M}$, es decir $0.82 \mathrm{\mu gmL}^{-1}$. De igual manera, a partir de los 60 minutos, la concentración del efluente se mantiene prácticamente constante en un valor de $2.0 \times 10^{-6} \mathrm{M}$, que corresponde a $0.47 \mathrm{\mu gmL}^{-1}$, una concentración pequeña en comparación con la concentración inicial de $12 \mu \mathrm{gmL}^{-1}$.

\section{CONCLUSIONES}

Se estudian dos muestras de carbón activado, una preparada a partir de carbón mineral activado con vapor de agua a $1123 \mathrm{~K}$ y otra comercial marca Merck, que se modifican químicamente para aumentar el contenido de grupos ácidos totales superficiales y se verifican cambios en la capacidad que tienen de retener carbamazepina desde soluciones séricas; los resultados obtenidos para el área superficial se encuentran en un rango entre 576 y $1025 \mathrm{~m}^{2} \mathrm{~g}^{-1}$ y los volúmenes de poro son proporcionales. La superficie química de los carbones activados se modifica por medio de soluciones de ácido nítrico al 40 y 65 por ciento y se obtiene en todos los casos un aumento en el contenido de grupos ácidos que varía entre 0.36 y $0.54 \mathrm{meqg}^{-1}$.

Se determina la variación en la entalpía de inmersión de las series de carbones activados en suero y en soluciones séricas de carbamazepina y se obtienen resultados entre 28 y $64 \mathrm{Jg}^{-1}$, con los valores más altos para el carbón activado mineral modificado con solución de ácido nítrico al 40 por ciento, CAmi40, y las cantidades adsorbidas de carbamazepina varían entre 0.096 y $0.111 \mathrm{mgg}^{-1}$. Los carbones activados obtenidos a partir de carbón mineral presentan los valores de variación de entalpía más altos, lo que indica que la interacción entre el soluto y estos sólidos es mayor y que está influenciada tanto por factores físicos como químicos de la superficie.

Se estudia el cambio de concentración de soluciones que pasan a través del carbón activado en función del tiempo, durante períodos de dos horas, obteniendo los mayores resultados en la retención para los carbones activados CAmi40, CAmi 65 y CAmk65; este resultado refuerza la información suministrada en la determinación de la variación en la entalpía neta de la carbamazepina con los sólidos que permite observar una mayor interacción con el carbón activado obtenido de carbón mineral y modificado químicamente con solución de ácido nítrico del $40 \%$. 


\section{AGRADECIMIENTOS}

Los autores agradecen al convenio marco existente entre la Universidad Nacional de Colombia y la Universidad de Los Andes. Al igual, al fondo de investigación de proyecto semilla de la Facultad de Ciencias de la Universidad de Los Andes.

\section{REFERENCIAS}

Boehm, H.P.; Advances in Catalysis. Eley, D.D.H.Pines., P.B.Weisz, Eds. Academic Press. New York. 192 (1966).

Cartula, A. y otros cuatro autores; Adsorption of Substituted Phenols on Activated Carbon. J. Colloid and Interface Science, 24, 528 (1998).

Chen, X. y otros cinco autores; Mechanisms of surfactant adsorption on non- polar, air oxidized and ozone treated carbon surfaces. Carbon 41, 1489 (2003).

Cyted; Taller Iberoamericano sobre adsorbentes para la protección ambiental, Red Temática V.F., Guanajuato, México (2004).

Dastgheib, S.A. y T. Karanfil, Adsorption of oxygen by heat treated granular and fibrousactivated carbons. J. Colloid. Int. Sience, 1 (2004).

Gaudreault, P.; Activated Charcoal Revisited. Clinical Pediatric Emergency Medicine. 6 ,76 (2005).

Giraldo, L., J.C. Moreno y J.I. Huertas; Heats Conduction Micro-Calorimeter With Metallic Reaction Cells. Instrumentation Science \& Technology, 30 (2), 177 (2002).

Krenzelok, E. P.; New Development in the Therapy of Intoxications. Toxicology Letters, 127 (1-3) 299 (2002).

Ladino, Y., L. Giraldo y J.C. Moreno; Calorimetric study of the immersion heats of lead (II) and chromium (VI) from aqueous solutions of colombian coffee husk. Journal Thermal Analisys and Calorimetry, 81, 435 (2005).

$\mathrm{Li}$, Y.H. y otros siete autores; Competitive adsorption of $\mathrm{Pb}^{2+}, \mathrm{Cu}^{2+}$ and $\mathrm{Cd}^{2+}$ ions from aqueous solutions by multiwalled carbon nanotubes. Carbon, 41,2787 (2003) .

Lopez-Ramon, M.V., F. Stoeckli, C. Moreno-Castilla y F. Carrasco-Marín; On the Characterization of Acidic and Basic Surface Sites on Carbons by Various Techniques. Carbon 37, 215 (1999).

Moreno-Castilla, C; Adsorption of organic molecules from aqueous solutions on carbon materials. Carbon. 42 , 83 (2004).

McGuire, M.J.e I.H. Suffet; Treatment of water by granular activated carbon. Ed. American Chemical Society. Washington D.C. 29 (1983).

Neuvonen, P.J. y E. Clonen; "Effect of Activated Charcoal on Adsorption and Elimination of henobarbitone, Carbamazepine and Phenylbutazone in Man“; European Journal of Clinical Pharmacology. 17, 51 (1980).

Radovic, L.R., C. Moreno-Castilla y J. Rivera-Utrilla; Carbon Materials as Adsorbents in Aqueous Solutions. In Chemistry and Physics of Carbon. A Serie of Advances. pp 293 Ed Marcel Dekker. New York. (2000).

Raymundo-Piñero, E., D. Cazorla-Amorós y A. Linares-Solano; The role of different nitrogen functional groups on the removal of SO2 from flue gases by $\mathrm{N}$-doped activated carbons powders and fibres. Carbon 41, 1925 (2003). 
Rodríguez-Reinoso, F.; Activated carbon: structure, characterization, preparation and applications. En: Introduction to carbon technologies. Universidad de Alicante (Publicaciones). Alicante, España. 60 (1998).

Shin, S., J. Jang, H. Yoon e I. Mochida; A study on the effect of heat treatment on functional groups of pitch based activated carbon fiber using FTIR. Carbon 35,1739 (1997).

Suwalsky, M., S. Mennickent, B. Norris y H. Cardenas; The antiepilectic drug carbamazepineaffects sodium transport in toad epithelium. Toxicology in vitro. In press. (2006).

Tayel, S.A., I.I. Saliman y D. Louis ; Improvement of dissolution properties of carbamazepine trough application of the liquid solid tablet technique. Eur. J. Pharm. Biopharm., 2007, doi: 10.1016/jpb.2007.09.003

Tian, F. y otros siete autores; Visualizing the conversion of carbamazepine in aqueous suspension with and without the presence of excipients: A single crystal study using SEM and Raman microscopy. Eur. J. Pharm. Biopharm., 64 (3), 2006, 326-335 
